\title{
Impact of snow variability on the Swiss winter tourism sector: implications in an era of climate change
}

\author{
Camille Gonseth
}

Received: 10 May 2012 / Accepted: 13 February 2013 / Published online: 15 March 2013

(C) Springer Science+Business Media Dordrecht 2013

\begin{abstract}
With its numerous mountain regions and its well developed winter tourism infrastructures, Switzerland is a country whose tourism sector is known to be sensitive to snowpack variability. With climate change-which is predicted to have negative impacts on snow depths and duration - the need for accurately assessing the sensitivity of winter tourism consumption to changing snow conditions is reinforced. Taking advantage of newly available data on visitation rates at Swiss ski areas, we analyze the effect of snow conditions on skier visits using standard panel data regression techniques. We assume the magnitude of this effect to be conditional on the level of snowmaking investments. Higher snowmaking investments should lead to a lower sensitivity. Our results validate this hypothesis and also shed light on the competitive interactions between lower and higher lying ski areas located in the same tourism region. In fact, our results show that better snow conditions in the former reduces visitation rates in the latter. Eventually, we find that ski areas benefiting from sunny conditions tend to have, ceteris paribus, more skier visits. This suggests additional impacts if climate change were to modify sunshine duration in mountain regions.
\end{abstract}

\section{Introduction}

Climate change is a central issue for the Swiss winter tourism sector. Ski areas are or will be impacted in multiple ways by climate change. However, one crucial impact is related to deteriorating snow conditions. So far, many studies have analyzed the link between snowpack and climate conditions for Switzerland. Some

C. Gonseth $(\bowtie)$

Research Group on the Economics and Management of the Environment (REME),

École Polytechnique Fédérale de Lausanne (EPFL), Station 16, 1015 Lausanne, Switzerland

e-mail: camille.gonseth@epfl.ch 
researchers investigated past data and trends (Scherrer and Appenzeller 2006; Marty 2008; Serquet et al. 2011) while some explored future scenarios (Beniston et al. 2003; Uhlmann et al. 2009). These studies demonstrate that snowpack amount and duration already decreased, particularly since the end of the 1980's (Marty 2008). In the future, snow conditions will most probably continue to deteriorate. This will further impact the ski industry supply (König and Abegg 1997; Elsasser and Messerli 2001; Elsasser and Bürki 2002; Abegg et al. 2007). In particular, an OECD study (Abegg et al. 2007) looked at the consequences of a 1,2 , and $4{ }^{\circ} \mathrm{C}$ warming in the Alps. It found that 159 out of 164 Swiss ski areas are snow-reliable under current climatic conditions. The number will drop to respectively 142, 129, and 78 with the aforementioned increases in temperature. However, local scale modeling studies conducted in other ski regions (mainly in North America), which derive ski season length and snowmaking requirements under a warmer climate, consistently point to more moderate impacts (Scott et al. 2012). The strength of these studies compared to the previous ones is that they take into account snowmaking, whose increasing production and capacity improvement could significantly contribute to maintaining the ski season length (Scott et al. 2003, 2008; Steiger 2010).

Comparing winter seasons that are representative of current and future climatic conditions has been another way to estimate climate change impacts on the ski tourism industry (Dawson et al. 2009; Steiger 2011). As the analogue approach relies on observed data to predict future climate change impacts, it has the advantage to include many (potentially all) of the currently available, generally short-term adaptation possibilities on both the supply and demand sides. In the USA's Northeast region, this approach has provided lower impact estimates compared to those found using the modeling based approach (Dawson et al. 2009).

Another strand of the literature has more specifically dealt with potential and/or actual behavioral adaptations on the demand side (Behringer et al. 2000; Hamilton et al. 2007; Unbehaun et al. 2008; Shih et al. 2009; Pickering et al. 2010; Dawson et al. 2011; Töglhofer et al. 2011). Methodologically, these studies rely either on surveys of skiers or on quantitative analyses based on regression techniques. In the former case, results indicate that skiers would very probably move to more snow-reliable ski areas and ski less often. In the latter case, Töglhofer et al. (2011) for instance analyzed 185 Austrian ski resorts between 1973 and 2006 in order to quantify past demand changes due to short-term climate variability. They estimated an overall change of $0.6-1.9 \%$ in overnight stays with a one standard deviation change in snow conditions. These figures obtained for overnight stays probably underestimate the effect on skier visits as the latter are more sensitive to snow conditions than the former (Steiger 2011). Also potentially important for visitation rates at ski areas are snow conditions in skiers' areas of origin ("backyard hypothesis"). However, two studies, which both used detailed daily data from two individual ski areas, have provided conflicting evidence about this effect (Hamilton et al. 2007; Shih et al. 2009).

Studies investigating current relationships between weather conditions and ski participation are meaningful in the context of climate change as they are an important prerequisite for understanding the likely impacts of projected climate change on the ski industry. However, it is clear from the literature review that only few empirical researches have tackled so far the quantitative effect of snow conditions on ski area visitation. In the present article, we analyze the effect of snow and weather conditions on skier visits using linear panel regression models. The analysis is based on a panel of 74 Swiss ski areas tracked over the 2005/06 to 2008/09 winter seasons. 
The article is structured as follows: Section 2 presents and describes the method and data employed. Especially, we discuss ways by which we tried to obtain nonbiased estimates of the natural snow sensitivity of skier visits at a given ski area. Section 3 presents the results and discusses the role of snowmaking investments to reduce this sensitivity. Eventually, Section 4 offers some concluding remarks.

\section{Methodology and data}

\subsection{Regression model}

In order to assess the importance of natural snow conditions for the economies of Swiss alpine regions, we build a linear model of the natural logarithm of skier visits $\ln \left(S V_{i t}\right)$, which is a common measure of the number of visits at a ski area. Our fully specified model is the following:

$$
\begin{aligned}
\ln \left(S V_{i t}\right)= & \beta_{0}+\beta_{1} \cdot S D_{i t}+\beta_{2} \cdot A R T_{i t}+\beta_{3} \cdot\left(S D_{i t} \cdot A R T_{i t}\right) \\
& +\gamma^{\prime} \mathbf{X}_{\mathbf{i t}}+\mu_{i}+\delta_{t}+\varepsilon_{i t}
\end{aligned}
$$

where $S D_{i t}$ is the number of "skiable days" measured at ski area $i$ during the winter season $t, A R T_{i t}$ is the percentage of the kilometers of ski runs that can be artificially snowed in ski area $i$ and winter season $t, \mathbf{X}_{\mathbf{i t}}$ is a vector of control variables at the ski area $\mathrm{x}$ season level, $\mu_{i}$ is a ski area fixed effect, $\delta_{t}$ a winter season fixed effect, and $\varepsilon_{i t}$ is the idiosyncratic error term. A key feature of the model is the presence of the term $S D_{i t} \cdot A R T_{i t}$, which is the interaction term between the natural and artificial snow variables. This allows to take into account the fact that the short-term snow sensitivity of skier visits should normally decrease as the level of snowmaking investments increases. In fact, our main interest does not lie in estimating a single population regression coefficient. Rather, we are interested in estimating ME, the marginal effect of the number of skiable days, which is equal to:

$$
\operatorname{ME}\left(A R T_{i t}\right)=\left.\frac{\partial \ln \left(S V_{i t}\right)}{\partial S D_{i t}}\right|_{A R T_{i t}}=\beta_{1}+\beta_{3} \cdot A R T_{i t}
$$

This marginal effect has the following standard deviation:

$$
\sigma_{M E\left(A R T_{i t}\right)}=\sqrt{\operatorname{var}\left(\beta_{1}\right)+A R T_{i t}^{2} \cdot \operatorname{var}\left(\beta_{3}\right)+2 \cdot A R T_{i t} \cdot \operatorname{cov}\left(\beta_{1}, \beta_{3}\right)}
$$

Estimates of the short-term snow sensitivity of skier visits can easily be flawed due to omitted variable bias. This is the reason why the model includes a vector of control variables as well as fixed effects. It is advantageous to think of the vector of control variables as being made of three subsets of variables that describe respectively tourism infrastructures, ski area characteristics that are correlated with altitude and snow and weather conditions outside a ski area. All these three subsets of variables help mitigating the omitted variable bias problem. First, it seems reasonable to 


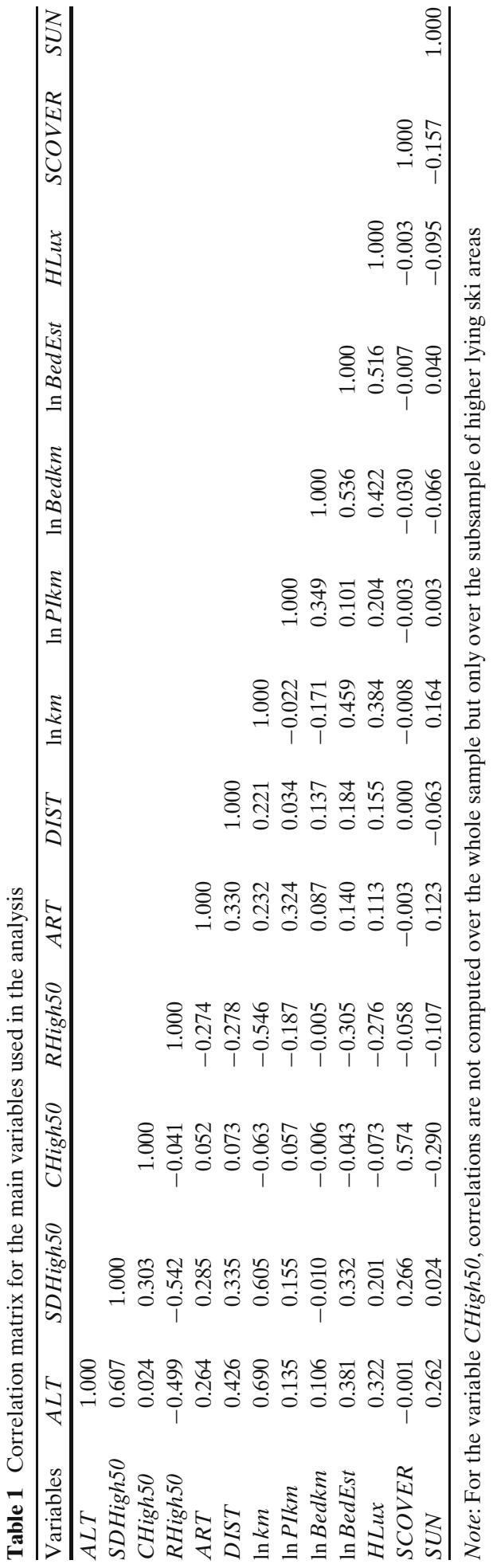


think that ski tourism infrastructures have historically developed in places with good snow conditions. Consequently, we expect the omission of this link to lead to an overestimation of the natural snow effect. As snow conditions improve with altitude, other ski area characteristics that are linked to altitude and that influence skier visits should also be controlled. As deduced from the correlations of Table 1, accessibility and sunshine are two examples of characteristics that are correlated with altitude. ${ }^{1}$ Another important factor changing with altitude and influencing visitation rates is the intra-seasonal distribution of skiable days. At higher altitudes, ski operators tend to have longer operation periods with a greater proportion of skiable days in November and April, two months that are rather marginal in importance for their business. Therefore, one should control for the intra-seasonal distribution to avoid underestimating the effect of good snow conditions. Eventually, it has been sometimes empirically tested in the literature that weather and snow conditions outside a ski area could also affect its visitation rate (Hamilton et al. 2007; Shih et al. 2009). These conditions should be controlled so that we could be a little more confident that our estimates of the snow sensitivity of skier visits represent the pure effect of improving snow conditions at the ski area itself. The detail and description of the variables that are included in the vector $\mathbf{X}_{\mathbf{i t}}$ are given in Section 2.2.

Regarding the fixed effects that are included in the model, it allows to extend the "control" strategy to non-observed, generally difficult-to-measure variables that are either ski area or time-invariant. Reputation, landscape's beauty or slopes orientation are examples of time-invariant characteristics of ski areas. The aim of adding winter season fixed effects is mainly to control for macroeconomic conditions (exchange rates, economic situation) that affect the tourism demand for Swiss winter destinations.

\subsection{Data description and source}

Skier visits data In this research, we investigate the impact of snow and weather conditions on skier visits. Vanat (2012) defines a skier visit as being generated by "one person visiting a ski area for all or any part of a day or night for the purpose of skiing, snowboarding, or other downhill sliding”. Since a couple of years, he collects annual data on several operational indicators directly from a large sample of ski operators. The internal consistency of these data is carefully checked so that a reliable skier visits statistic is produced annually. ${ }^{2}$ Thanks to this statistic, we were

\footnotetext{
${ }^{1}$ At the ski area level, the variable depicting altitude $(A L T)$ is derived by taking the highest value among the altitudes of the transport facilities' upper stations. Throughout the paper, we define higher lying ski areas as ski areas whose maximum altitude lies above $2,500 \mathrm{~m}$. The chosen threshold is somewhat arbitrary but has the advantage to split our sample of ski areas in two subsamples of equal size.

${ }^{2}$ It is acknowledged that the measurement of skier visits is not completely homogenous within the surveyed companies. However, this should not cause estimation problems. First, it is uncertain whether a change in the counting method introduces a systematic bias in the number of skier visits, nor whether the use of a particular method is correlated with other characteristics of ski areas. Moreover, it is likely to be a time-invariant characteristic of ski areas over our sample period. This facilitates its control through proper estimation methods.
} 
able to gather data on skier visits for a sample of approximately 70 Swiss ski areas across the winter seasons 2005/06 to 2007/08 but only for 62 ski areas during the 2008/09 winter season. The relative large change in the sample's size across the years has to do with the fact that the skier visits statistic is not mandatory. Our panel dataset is therefore an unbalanced one. It has been built to satisfy methodological requirements (at least two consecutive years of data per ski area) but was limited to four winter seasons due to both data and time constraints. The sample of skier visits is presented in Fig. 1.

For the four winter seasons under review, features of the skier visits distribution can be assessed from Fig. 1. In general, the median lies around 200,000 skier visits whereas the interquartile range (IQR) is roughly equal to 300,000 skier visits. With its relatively strong reduction in skier visits $(-15.3 \%$ compared to the 10 -year average 2000/01-2009/10), the snow-poor 2006/07 winter season is visible from the boxplot. It is interesting to note that our sample therefore includes information on how ski operators and their customers have responded to this record warm winter season. However, the most striking feature of the plot are the shapes of the distributions which are heavily skewed towards high values. With its strictly positive nature and asymmetric distribution, the dependent variable is a good candidate for transformation with the natural logarithm. As a result of this transformation, model coefficient estimates will be used to compute relative rather than absolute changes in the dependent variable. We are more confident dealing with relative impact estimates as our sample includes a broad spectrum of ski areas, from small sized ski areas to some of the world's most renowned ones.

Snow data For the purpose of our analysis, we have built two alternative measures of snow conditions at ski areas. Both are derived from data provided by the Swiss National Tourist Office (SNTO). In order to publicize them on its website, the SNTO asks ski operators to measure snow heights at three different elevations (including at the lowest and highest altitudes of ski areas) on a day-to-day basis. Strict rules set by the SNTO imply that measurement stations should be located next to the ski area

Fig. 1 Boxplots of gathered skier visits values for the winter seasons 2005/06 to 2008/09. Boxes are delimited by the quartiles and the line in their center represents the median. The interquartile range (IQR) is given by the length of a box. Whiskers indicate the upper and lower observations within 1.5 times the IQR beyond the 25 th and 75 th percentile values. Observations beyond that range (outliers) are individually marked with open black circles

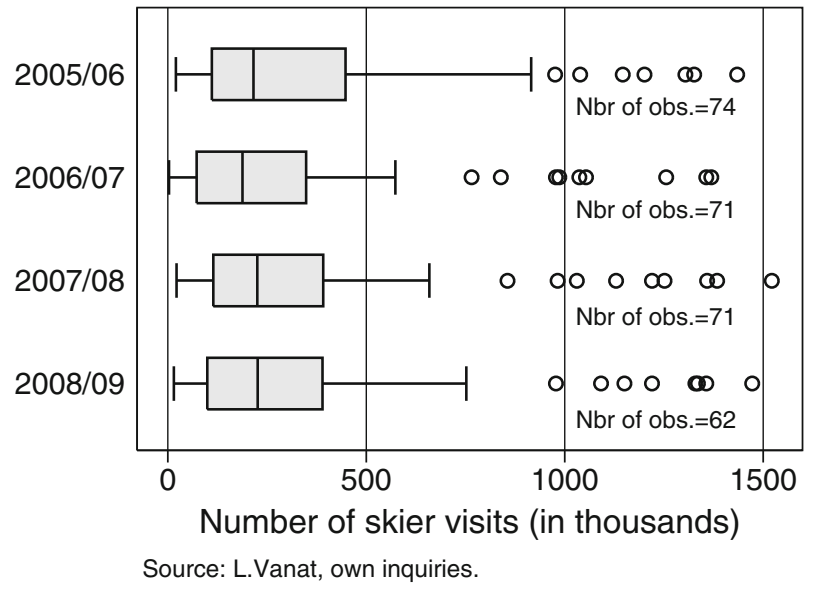

Source: L.Vanat, own inquiries. 
with no influence of machine-made snow on measured snow depths. We are aware that self-reporting casts doubt on the quality of these data. However, controls and sanctions exist to avoid systematic misreporting.

For a given winter season, our first measure of snow conditions gives the number of days with at least $30 \mathrm{~cm}$ of natural snow in the lower part of the ski area (SDLow30), whereas the second one indicates the number of days with at least 50 $\mathrm{cm}$ of natural snow in the higher part of the ski area $(S D H i g h 50)$. Both variables are an attempt to measure the number of "skiable days", i.e. the number of days which satisfy the minimum snow depth requirement for skiing. ${ }^{3}$ The two variables depicting snow conditions are tightly linked for ski areas located at higher altitudes as the sample correlation approaches $1(\rho=0.92)$. Discrepancies from a perfect linear relationship are slightly more pronounced for lower lying ski areas $(\rho=0.82)$. We expect more skiable days to lead to more skier visits.

Based on these two measures of snow conditions, we have also built another set of two variables that are specific to higher lying ski areas. These variables are equal to the weighted average of skiable days across the lower lying ski areas that are located in the same tourism region. Using SDLow30 as the measure of skiable days leads to the variable CLow30 whereas the variable CHigh50 is obtained when using SDHigh50. Weights depend upon the transport capacity installed at each lower lying ski area. The tourism regions definition has been chosen according to the one provided by the Swiss Tourism Federation, which divides Switzerland into 13 distinct areas. Within a tourism region, we expect better snow conditions in lower lying ski areas to negatively impact visitation rates at higher lying ones.

Eventually, the intra-seasonal distribution of skiable days is measured by computing the fraction of skiable days that take place during the months of December to March. This fraction is denoted RHigh50 (RLow30) in case the variable SDHigh50 (SDLow30) is used to derive it. This ratio should be positively correlated with the number of skier visits.

Weather data Both weather conditions at ski areas and in the lowlands are considered in this study. Based on data from the SNTO, we computed the fraction of sunny days over the total number of operation days $(S U N)$ as our measure of weather conditions at the ski area. We also derived three other measures from the same dataset but only used them as alternatives to perform robustness checks. These measures are the following: fraction of sunny and slightly cloudy days over the total number of operation days (SUNCLOUD), and the fractions over the total number of weekend days during the operating season with either one or the other of the two aforementioned conditions (WESUN, WESUNCLOUD). Sunny days are defined as days without clouds, whereas partly cloudy days are defined as days with less than $3 / 8$ of the sky covered by clouds. The choice to focus on weekends comes from the expectation that day trippers mainly ski during these days. We expect good weather conditions at the ski area to have a positive impact on the number of skier visits and

\footnotetext{
${ }^{3}$ It is common to find an operational threshold of $30 \mathrm{~cm}$ in the literature (Scott et al. 2003; Steiger 2010). We assume that more snow is needed for skiing at higher altitudes because of the more rugged terrain.
} 
therefore a positive associated coefficient. In relation to weather conditions in the lowlands, two alternative measures were derived using data from the Swiss Federal Office of Meteorology and Climatology. For the winter seasons 2005/06 to 2008/09, we first summed the monthly numbers of days with snow cover and the monthly average temperature (2 m above ground) deviations from norm (1961-1990) at four meteorological stations located in the main urban centers (Zürich, Bern, Basel and Geneva). We then took the average across meteorological stations of these sums in order to obtain two variables that vary only according to the time dimension (SCOVER and DEVTMEAN).

Other data and the correlation matrix The last set of variables describes some additional characteristics of ski areas such as accessibility, the total length of ski runs, hotel accommodation supply and the level of investments in both snowmaking and transport facilities. We define accessibility as the time taken by car to reach a given ski area from the nearest urban center. Accordingly, we constructed a binary variable DIST that takes the value 1 whenever more than $90 \mathrm{~min}$ are needed by car for that journey and 0 otherwise. The accessibility data were obtained by using the online cartography website, http://fr-ch.mappy.com (accessed: 15/10/12), which allows computing car travel time between destinations. We expect remoteness and skier visits to be negatively correlated. The variable $\ln \mathrm{km}$ is the natural logarithm of the total length of ski runs (in kilometers). It controls for scale effects meaning that the sign of its associated coefficient should be positive. Based on data from the Swiss Federal Statistical Office, we use several variables to describe the supply characteristics of hotel accommodation: the average number of available beds during the winter season per $\mathrm{km}$ of slopes $(\ln B e d \mathrm{~km})$, the average number of beds per establishment (ln BedEst), and the percentage of beds in 4-5 stars hotels (HLux). The first two of these variables are transformed with the natural logarithm. Taken altogether, their aim is to control for the hotel accommodation's size and structure at a given ski area. Common sense suggests that skier visits should be positively correlated with the number of hotel beds which would translate into a positive regression coefficient on the variable $\ln B e d \mathrm{~km}$. We make no guess as to the sign of the coefficients on the other two variables. Our measure of artificial snow production capacity $(A R T)$ represents the percentage of the ski slopes' length that can be snowed using the available snowmaking facilities. This information was gathered from different sources including the direct questioning of ski operators. Eventually, information on transport infrastructures at ski areas was either obtained from the Swiss Federal Office for Spatial Development or, for more recent information, from the Swiss Ski Operators Association. The transport capacity related variable is computed in three steps. The first step amounts to multiply a ski lift, chair lift or cable car's transport capacity (persons/hour) with its difference in height (given in $\mathrm{km}$ ). We then sum this value over all facilities located at a given ski area and divide that sum by the total length of ski runs. We end up taking the natural logarithm to obtain the variable $\ln P I \mathrm{~km}$. We expect the regression coefficient to be positive for this variable.

In Table 1, we provide the correlation matrix for the main explanatory variables used in this paper. We already used some of the information that it contains in Section 2.1 and we will again refer to it in Section 3.2 in order to shed light on some of our results. 


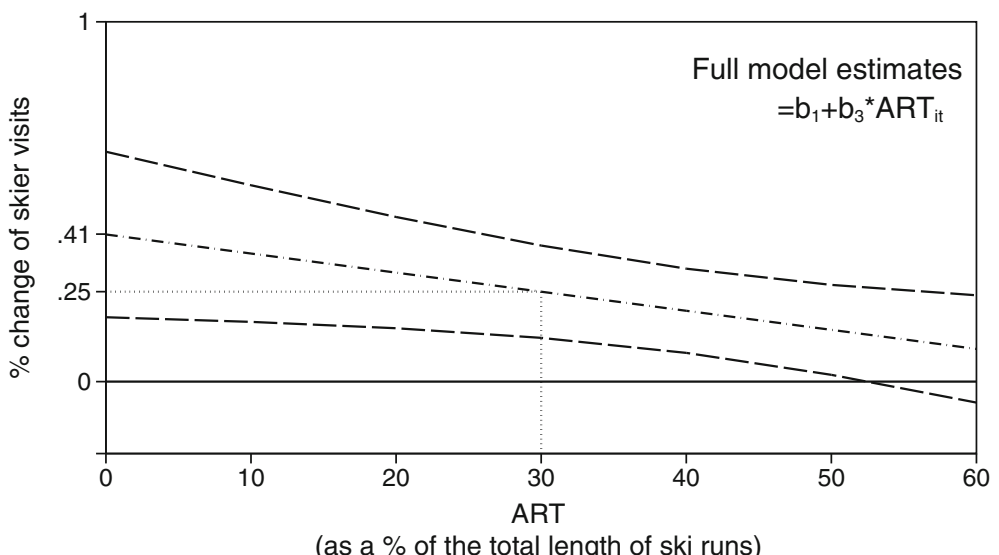

Fig. 2 Marginal effects of skiable days on skier visits depending on artificial snow capacity with $95 \%$ confidence intervals. Values necessary to graph marginal effects and confidence intervals are based on the fixed effects estimates of Eq. 1. The average coverage of snowmaking is roughly equal to $30 \%$ in our sample. At this value of the variable $A R T$, the marginal effect of one additional skiable day on seasonal skier visits is estimated to be equal to $0.25 \%$

\section{Results}

\subsection{Marginal effects}

To compute the marginal effects of skiable days on seasonal skier visits, we use the results obtained when estimating Eq. 1's parameters with the fixed effects estimator. ${ }^{4}$ More precisely, these results are used to compute marginal effects and their standard error according to the Eqs. 2 and 3 shown above. For a snowmaking percentage coverage ranging from 0 to $60 \%$, these estimated values then serve as the basis for building Fig. 2.

As can be seen, the marginal effect is significantly different from zero and positive on a continuous interval of the variable $A R T$ ranging from 0 to roughly $50 \%$. This interval encompasses most of the "real-world", observed values contained in our sample as the upper quartile of the variable $A R T$ is equal to $37 \%$ for the 2008/09 winter season. Figure 2 makes also clear that the marginal effect of natural snow conditions on skier visits decreases with the level of snowmaking investments. This proves that snowmaking reduces the short-term sensitivity of skier visits to natural snow conditions. Consequently, snowmaking shows significant potential for smoothing the inter-annual variability of skier visits caused by the natural variation in snow conditions. Without snowmaking, the mean value of the marginal effect is equal to $0.41 \%$. With a $30 \%$ coverage corresponding to the average value of $A R T$ across our panel dataset, the marginal effect shrinks to $0.25 \%$ (i.e. a $39 \%$ reduction).

\footnotetext{
${ }^{4}$ Since it controls for time-invariant ski area characteristics, we consider results obtained with the fixed effects estimator as our benchmark. The fact to control for these characteristics has significant effects on coefficient estimates (cf. the result from the Hausman test, reported in Table 2, which compares estimation outcomes from the random and fixed effects estimators. Its p-value smaller than 0.05 indicates that we should prefer the latter estimator to the former).
} 
Table 2 Regression estimates obtained with different models and estimators

\begin{tabular}{|c|c|c|c|c|c|}
\hline $\begin{array}{l}\text { Dep. Var.: } \\
\ln (\mathrm{SV})\end{array}$ & $\begin{array}{l}\text { (1) } \\
\text { Pooled } \\
\text { OLS }\end{array}$ & $\begin{array}{l}\text { (2) } \\
\text { Pooled } \\
\text { OLS }\end{array}$ & $\begin{array}{l}\text { (3) } \\
\text { First- } \\
\text { diff. }\end{array}$ & $\begin{array}{l}(4) \\
\text { Random } \\
\text { effects }\end{array}$ & $\begin{array}{l}(5) \\
\text { Fixed } \\
\text { effects }\end{array}$ \\
\hline SDHigh50 & $\begin{array}{l}0.0036^{*} \\
{[0.0020]}\end{array}$ & $\begin{array}{r}0.0056 * * * \\
{[0.0020]}\end{array}$ & $\begin{array}{r}0.0043^{* * * *} \\
{[0.0010]}\end{array}$ & $\begin{array}{r}0.0040 * * * \\
{[0.0013]}\end{array}$ & $\begin{array}{r}0.0041 * * * \\
{[0.0012]}\end{array}$ \\
\hline$A R T$ & $\begin{array}{l}0.0093 \\
{[0.0063]}\end{array}$ & $\begin{array}{l}0.0078^{*} \\
{[0.0043]}\end{array}$ & $\begin{array}{c}0.0082^{* *} \\
{[0.0038]}\end{array}$ & $\begin{array}{l}0.0088^{* *} \\
{[0.0045]}\end{array}$ & $\begin{array}{l}0.0096 * * \\
{[0.0044]}\end{array}$ \\
\hline SDHigh50 $\cdot A R T$ & $\begin{array}{r}-0.000055 \\
{[0.000048]}\end{array}$ & $\begin{array}{r}-0.000061^{*} \\
{[0.000034]}\end{array}$ & $\begin{array}{c}-0.000048^{* *} \\
{[0.000021]}\end{array}$ & $\begin{array}{r}-0.000052^{*} \\
{[0.000027]}\end{array}$ & $\begin{array}{r}-0.000053^{* *} * \\
{[0.000025]}\end{array}$ \\
\hline CHigh50 & $\begin{array}{l}0.00022 \\
{[0.00076]}\end{array}$ & $\begin{array}{c}-0.00083 * * * \\
{[0.00024]}\end{array}$ & $\begin{array}{l}-0.0013 \\
{[0.00086]}\end{array}$ & $\begin{array}{c}-0.00060 \\
{[0.00051]}\end{array}$ & $\begin{array}{r}-0.0014 * * \\
{[0.00062]}\end{array}$ \\
\hline$S U N$ & $\begin{array}{r}-0.042 \\
{[0.25]}\end{array}$ & $\begin{array}{l}0.096 \\
{[0.13]}\end{array}$ & $\begin{array}{l}0.30^{* *} \\
{[0.12]}\end{array}$ & $\begin{array}{l}0.25^{* *} \\
{[0.12]}\end{array}$ & $\begin{array}{l}0.27 * * \\
{[0.12]}\end{array}$ \\
\hline DIST & $\begin{array}{r}-0.089 \\
{[0.10]}\end{array}$ & $\begin{array}{r}-0.065^{*} \\
{[0.036]}\end{array}$ & & $\begin{array}{c}-0.043 \\
{[0.099]}\end{array}$ & \\
\hline$D I S T \cdot S C O V E R$ & $\begin{array}{r}-0.00051 \\
{[0.0011]}\end{array}$ & $\begin{array}{l}0.0011 \\
{[0.00086]}\end{array}$ & $\begin{array}{c}-0.00051 \\
{[0.00068]}\end{array}$ & $\begin{array}{c}-0.00087 \\
{[0.00073]}\end{array}$ & $\begin{array}{c}-0.00055 \\
{[0.00069]}\end{array}$ \\
\hline RHigh50 & $\begin{array}{r}0.93 * * * \\
{[0.34]}\end{array}$ & $\begin{array}{r}1.38 * * * \\
{[0.38]}\end{array}$ & $\begin{array}{r}0.72 * * * \\
{[0.20]}\end{array}$ & $\begin{array}{r}0.68 * * * \\
{[0.22]}\end{array}$ & $\begin{array}{r}0.74 * * * \\
{[0.22]}\end{array}$ \\
\hline $\ln k m$ & $\begin{array}{c}1.04 * * * \\
{[0.077]}\end{array}$ & $\begin{array}{c}0.23 * * * \\
{[0.071]}\end{array}$ & $\begin{array}{l}0.098 \\
{[0.38]}\end{array}$ & $\begin{array}{l}1.10 * * * \\
{[0.073]}\end{array}$ & $\begin{array}{l}0.24 \\
{[0.36]}\end{array}$ \\
\hline $\ln P I k m$ & $\begin{array}{r}1.10^{* * * *} \\
{[0.12]}\end{array}$ & $\begin{array}{l}0.17^{* *} \\
{[0.070]}\end{array}$ & $\begin{array}{l}0.18 \\
{[0.16]}\end{array}$ & $\begin{array}{r}0.92 * * * \\
{[0.13]}\end{array}$ & $\begin{array}{l}0.17 \\
{[0.17]}\end{array}$ \\
\hline $\ln \mathrm{Bedkm}$ & $\begin{array}{c}-0.084 \\
{[0.065]}\end{array}$ & $\begin{array}{l}0.014 \\
{[0.016]}\end{array}$ & $\begin{array}{l}0.029 \\
{[0.072]}\end{array}$ & $\begin{array}{l}0.0074 \\
{[0.047]}\end{array}$ & $\begin{array}{l}0.069 \\
{[0.061]}\end{array}$ \\
\hline $\ln$ BedEst & $\begin{array}{l}0.23^{*} \\
{[0.13]}\end{array}$ & $\begin{array}{c}-0.053 \\
{[0.043]}\end{array}$ & $\begin{array}{c}-0.18 \\
{[0.16]}\end{array}$ & $\begin{array}{l}0.042 \\
{[0.11]}\end{array}$ & $\begin{array}{r}-0.25^{*} \\
{[0.15]}\end{array}$ \\
\hline HLux & $\begin{array}{l}0.0014 \\
{[0.0026]}\end{array}$ & $\begin{array}{l}0.0022 * * \\
{[0.0010]}\end{array}$ & $\begin{array}{r}-0.00023 \\
{[0.0011]}\end{array}$ & $\begin{array}{l}0.0017 \\
{[0.0017]}\end{array}$ & $\begin{array}{l}0.00069 \\
{[0.0015]}\end{array}$ \\
\hline$l \cdot \ln (S V)$ & & $\begin{array}{c}0.79 * * * \\
{[0.061]}\end{array}$ & & & \\
\hline Ski area FE & no & no & yes & no & yes \\
\hline Winter season FE & yes & yes & yes & yes & yes \\
\hline $\begin{array}{l}\text { Observations } \\
R^{2} \\
\text { Prob }>\text { chi } 2 \\
\quad(\text { Hausman test })\end{array}$ & $\begin{array}{l}228 \\
0.913\end{array}$ & $\begin{array}{l}164 \\
0.979\end{array}$ & $\begin{array}{l}157 \\
0.635\end{array}$ & $\begin{array}{l}228 \\
0.91\end{array}$ & $\begin{array}{l}228 \\
0.596 \\
0.036\end{array}$ \\
\hline
\end{tabular}

Notes: Robust standard errors in brackets; Standard errors are clustered at the ski area level *Significant at the $10 \%$ level; **Significant at the $5 \%$ level; ***Significant at the $1 \%$ level

Eventually, rising the average coverage of snowmaking from 30 to $50 \%$ reduces further the marginal effect from 0.25 to $0.145 \%$.

\subsection{Further results}

In this subsection, we report results of robustness checks and comment them. Doing so, we focus our discussion on estimates of specific model coefficients that are worth of interest with climate change. Columns (1) and (3)-(5) of Table 2 present results obtained with four different estimators: the pooled OLS, first-difference, random 
and fixed effects estimators. They are standard tools for estimating linear panel data regression models. ${ }^{5}$ In addition, column (2) shows the results obtained when the lagged dependent variable $(l \cdot \ln (S V))$ is added to the model as an explanatory variable.

From Table 2, we can infer that our central result concerning the marginal effects of skiable days on seasonal skier visits is robust to a variety of different model specifications and estimators. In fact, estimates of the parameters on both $S D H i g h 50$ and SDHigh50 $\cdot A R T$, which are used to build the marginal effects, remain relatively constant across columns (1)-(5) of Table 2. Moreover, results obtained with the alternative measure of snow conditions are also very similar. At snowmaking percentage coverage values of 0,30 and $50 \%$, marginal effects computed with the fixed effects estimates are then equal to respectively $0.47,0.27$ and $0.13 \%{ }^{6}$

The coefficient on SCOVER, which is a measure of the winter weather conditions in the lowlands that only varies according to the time dimension, cannot be estimated because all of the models in columns (1)-(5) include winter season fixed effects. However, it is possible to estimate the coefficient on the interaction term $D I S T$. SCOVER. It has been added to test the hypothesis that wintry conditions in the lowlands mostly benefit to ski areas that are located near densely populated agglomerations. In Switzerland, these ski areas are mostly regional ski destinations. The results shown in Table 2 do not validate this hypothesis since none of the regressions estimates a significantly negative coefficient on the interaction term. ${ }^{7}$ Another issue with these weather conditions in the lowlands is that their effects could be partially captured by the variable CHigh50. As explained in Section 2.2, this latter variable describes for a given ski area whose maximum altitude lies above 2,500 $\mathrm{m}$ the snow conditions in the lower lying ski areas within the same tourism region. Given this definition, we would expect CHigh50 to be correlated with SCOVER, an intuition that is confirmed by the sample correlation coefficient's value of 0.574 given in Table 1. Consequently, the detrimental impacts of good snow conditions in lower lying ski areas for the higher located ones could be underestimated by our estimates. Using the result from the fixed effects estimator, we predict that an increase of $\mathrm{CHigh} 50$ by one skiable day reduces the number of skier visits by $0.14 \%$ on average in the associated higher located ski areas.

Regarding weather conditions at the ski areas themselves, we found a positive and significant effect of sunny conditions on skier visits with the first-difference, random and fixed effects estimators. We can deduce from the parameter estimate obtained with the fixed effects estimator that skier visits are increased by $0.27 \%$ when the percentage of sunny days across the winter season increases from $1 \%$. No significant changes result from using SUNCLOUD, WESUN or WESUNCLOUD instead of $S U N$ in the regressions.

\footnotetext{
${ }^{5}$ We refrain from showing estimation results from a dynamic panel data model with fixed effects using the Arellano-Bond estimator. In the context at hand, it makes little sense to use this estimator since only four time periods are available. This gives rise to only few lagged values of skier visits that can be used as instruments whereas skier visits are likely to be strongly correlated from one year to the next. This looks like a setting where we could hardly get anything else than weak instruments.

${ }^{6}$ Detailed estimation results of these regression runs are available from the author on request.

${ }^{7}$ Results do not change if we use DEVTMEAN rather than SCOVER as the variable depicting weather conditions in the lowlands.
} 
Eventually, parameter estimates for the remaining control variables generally show the expected sign and several of them are significantly different from zero. Nonetheless, a striking element is the fact that the variable ln Bedkm does not have a significantly positive effect on the number of skier visits. This could be the consequence of omitting the supply of holiday accommodation outside the hotel industry in our models, which may well be negatively correlated with the hotel accommodation supply once scale effects are controlled for. Para-hotel accommodation supply was not integrated in our analysis because of a lack of data. Note also that the firstdifference and fixed effects estimators, which use the variation of a variable across the time dimension to identify its effect on the dependent variable, have difficulties pinning down the effect of variables like $\ln \mathrm{km}$ or $\ln P I \mathrm{~km}$. This is due to the fact that the variability of these variables is mostly across ski areas.

\section{Conclusion}

The snowpack reduction and the reduced number of skiable days implied by climate change is expected to impact the ski industry in Europe. Since Swiss ski areas are located on average at higher altitude than those of neighboring countries, the ski industry is expected to be less affected in Switzerland than in the other European alpine countries. This does not mean though, that adaptation measures will not be required nor undertaken in Switzerland. As emphasized in Scott et al. (2008), adaptive capacity by ski areas will be, together with local climatic conditions, a crucial factor to determine who will survive in an era of climate change. In particular, underinvestment in snowmaking may result in significant competitive disadvantages. In fact, our results show that snowmaking facilities prove to be valuable under the current climate for reducing the short-term sensitivity of skier visits to the natural snow conditions. Compared to a ski area that has no snowmaking facilities, a ski operator that can ensure the presence of snow over $30 \%$ of the ski runs it operates (which is roughly the Swiss average) reduces the natural snow sensitivity of its skier visits by $39 \%$. These results also support the findings of the existing literature that a larger snowmaking coverage help reducing significantly the detrimental impacts of snow-poor winter seasons for individual ski areas (Steiger 2011).

With a relatively low level of snowmaking coverage on average, the Swiss ski industry seems to be in a position where it can still afford to further reduce its vulnerability to natural snow conditions. This potential is particularly interesting in case climate change increases the inter-annual variability of the number of skiable days. We can deduce, from our results, that an increase in the snowmaking percentage coverage from $30 \%$ of the total length of ski runs to $50 \%$ could counteract a $42 \%$ increase in the natural snow conditions' variability. However, this is only possible under the assumption that snowmaking will remain both technically and economically feasible in a future warmer climate.

While the reduction in snowpack is certainly a central impact, climate change could also modify visitation rates at ski areas through other channels. For instance, a milder weather in the lowlands during the winter or a change in sunshine duration at higher elevation sites could probably also modify the number of people going to the mountains for skiing. We found that sunny conditions at ski areas positively influence the number of skier visits but we could not identify the effect related to 
weather conditions in the lowlands because of a specification problem. As pointed out in the results section, the "backyard" effect of weather conditions in the lowlands could however be partly captured by the parameter on the variable describing snow conditions in the lower located ski areas. If it were the case, our estimates of this parameter would be upward biased meaning that we would tend to underestimate the negative effect for the higher lying ski areas of good operating conditions in the lower located ones. Despite this potential source of confusion, we found that poor snow conditions in lower lying ski areas do indeed increase the number of skier visits in higher lying ones. This is in line with the results of stated behavior surveys, including the one carried out in Switzerland (Behringer et al. 2000), as it suggests that some skiers move to more snow-reliable ski destinations in case of snow-poor winter seasons. As noted in several papers (Scott et al. 2008; Dawson and Scott 2010), this spatial substitution could occur under a warmer climate and benefit to ski areas that will remain operational. This effect is however uncertain in the long run since the most vulnerable ski areas (e.g. the lower lying ones) are generally highly valued by families and their closure may also prevent the renewal of generations of skiers (Scott et al. 2012).

Acknowledgements The author gratefully acknowledges the support of the Swiss Federal Office for the Environment as well as of the Swiss National Center of Competence in Research on Climate. The author also thanks Cecilia Matasci and Laurent Vanat for their help and Nicole Mathys, Philippe Thalmann and three anonymous referees for helpful comments.

\section{References}

Abegg B, Agrawala S, Crick F, de Montfalcon A (2007) Climate change impacts and adaptation in winter season. In: Agrawala S (ed) Climate change in the European Alps: adapting winter tourism and natural hazards management. Organisation for Economic Co-operation and Development (OECD), Paris, pp 25-60

Behringer J, Buerki R, Fuhrer J (2000) Participatory integrated assessment of adaptation to climate change in alpine tourism and mountain agriculture. Integr Assess 1:331-338

Beniston M, Keller F, Koffi B, Goyette S (2003) Estimates of snow accumulation and volume in the Swiss Alps under changing climatic conditions. Theor Appl Climatol 76:125-140

Dawson J, Scott D (2010) Systems analysis of climate change vulnerability for the US Northeast ski sector. Tour Hosp Plann Dev 7:219-235

Dawson J, Scott D, McBoyle G (2009) Climate change analogue analysis of ski tourism in the northeastern USA. Clim Res 39:1-9

Dawson J, Havitz M, Scott D (2011) Behavioral adaptation of alpine skiers to climate change: examining activity involvment and place loyalty. J Travel Tourism Market 28:388-404

Elsasser H, Bürki R (2002) Climate change as a threat to tourism in the Alps. Climate Res 20:253-257

Elsasser H, Messerli P (2001) The vulnerability of the snow industry in the Swiss Alps. Mt Res Dev 21:335-339

Hamilton L, Brown C, Keim BD (2007) Ski areas, weather and climate: time series models for New England case studies. Int J Climatol 27:2113-2124

König U, Abegg B (1997) Impacts of climate change on winter tourism in the Swiss Alps. J Sustain Tourism 5:46-59

Marty C (2008) Regime shift of snow days in Switzerland. Geophys Res Lett 35:L12501. doi:10.1029/ 2008GL033998

Pickering CM, Castley JG, Burtt M (2010) Skiing less often in a warmer world: Attitudes of tourists to climate change in an Australian ski resort. Geogr Res 48:137-147

Scherrer SC, Appenzeller C (2006) Swiss Alpine snow pack variability: major patterns and links to local climate and large-scale flow. Clim Res 32:187-199 
Serquet G, Marty C, Dulex JP, Rebetez M (2011) Seasonal trends and temperature dependence of the snowfall/precipitation day ratio in Switzerland. Geophys Res Lett 38:L07703. doi:10.1029/2011GL046976

Scott D, McBoyle G, Mills B (2003) Climate Change and the skiing industry in southern Ontario (Canada): exploring the importance of snowmaking as a technical adaptation. Clim Res 23:171181

Scott D, Dawson J, Jones B (2008) Climate change vulnerability of the US Northeast winter recreation-tourism sector. Mitig Adapt Strategies Glob Chang 13:577-596

Scott D, Gössling S, Hall MC (2012) International tourism and climate change. WIREs Clim Change 3:213-232

Shih C, Nicholls S, Holecek DF (2009) Impact of weather on downhill ski lift ticket sales. J Travel Res 47:359-372

Steiger R (2010) The impact of climate change on ski season length and snowmaking requirements in Tyrol, Austria. Clim Res 43:251-262

Steiger R (2011) The impact of snow scarcity on ski tourism: an analysis of the record warm season 2006/2007 in Tyrol (Austria). Tourism Rev 66:4-13

Töglhofer C, Eigner F, Prettenthaler F (2011) Impacts of snow conditions on tourism demand in Austrian ski areas. Clim Res 46:1-14

Uhlmann B, Goyette S, Beniston M (2009) Sensitivity analysis of snow patterns in Swiss ski resorts to shifts in temperature, precipitation and humidity under conditions of climate change. Int $\mathrm{J}$ Climatol 29:1048-1055

Unbehaun W, Pröbst U, Haider W (2008) Trends in winter sport tourism: challenges for the future. Tourism Rev 63:36-47

Vanat L (2012) International report on mountain tourism-overview of the key industry figures for ski resorts. http://www.vanat.ch/RM-world-report-2012.pdf. Accessed 15 October 2012 